\title{
Fibrin Sealant Patch for the Management of Intractable Bleeding From an LVAD Apex Cannula
}

\author{
Kiril Penov, Dejan Radaković, Seymur Karimli, Ivan Aleksić \\ Department of Thoracic and Cardiovascular Surgery, University Hospital Würzburg, Würzburg, Germany
}

\section{ABSTRACT}

Background: Intractable bleeding from the apical cannulation site of a left ventricular assist device (LVAD) is a dreaded complication.

Case report: A 52-year-old male suffering from dilative cardiomyopathy (DCM) with fixed pulmonary hypertension underwent reoperative LVAD implantation after previous mitral valve surgery. The patient underwent three rethoracotomies for bleeding from the apex cannulation site without achieving hemostasis. Conventional techniques and application of fibrin sealants and polymeric sealing devices did not fix the problem. The bleeding stopped after application of the EVARREST® Fibrin Sealant Patch (FSP), and he needed no further transfusions.

Conclusion: This patch might become a useful tool for intractable bleeding problems in LVAD surgery.

\section{INTRODUCTION}

Conventional methods of achieving hemostasis like sutures, cautery, clips, or energy-based coagulation depend on anatomical location, accessibility and type of tissue, among others. If these methods are ineffective, topical absorbable hemostats (TAHs) gain relevance. In case of severe bleeding, TAHs are less effective due to a lack of adhesion [Ding 2013]. The EVARREST ${ }^{\circledR}$ fibrin sealant patch (FSP) (Ethicon, Somerville, New Jersey, USA) consists of polyglactin (PG910), oxidized regenerated cellulose (ORC), human fibrinogen, and human thrombin. Several studies have demonstrated its effectiveness and safety in different tissue types and over a wide array of bleeding intensities [Fischer 2013; Koea 2015]. However, the experience with fibrin pads in cardiac surgery, by itself prone to major bleeding issues with full-heparinization, is very limited. In case of bleeding from the apex cannula of a left ventricular assist device (LVAD) difficult accessibility and anatomical location of the bleeding site

Received fune 25, 2021; accepted fuly 6, 2021.

Correspondence: Ivan Aleksić, MD, PhD, FETCS, Klinik fïr Thorax-, Herz- und Thorakale Gefäßchirurgie, Universitätsklinikum Würzburg, Oberdürrbacher Strasse 6, 97080 Würzburg, Germany, Telephone +49 931 201 33001, Fax +4993120133009 (e-mail: Aleksic_I@ukw.de) come into play. We describe the successful management of such a bleeding complication with the EVARREST ${ }^{\circledR}$ patch after conventional hemostatic measures failed.

\section{CASE REPORT}

A 52-year-old man with dilated cardiomyopathy and fixed pulmonary hypertension underwent LVAD implantation with the intent to reduce elevated pulmonary artery pressures and a pulmonary vascular resistance (PVR) of 8.3 Wood-Units. The patient had undergone prior mitral valve reconstruction and implantation of a biventricular cardioverter-defibrillator (CRT-ICD). A HeartWare (Medtronic, Dublin, Ireland) device was implanted to achieve transplant eligibility or as destination therapy, if the attempt to reduce PVR failed.

Concomitant tricuspid valve reconstruction with a $34 \mathrm{~mm}$ CE Physio Ring (Edwards Lifesciences, Irvine, CA, USA) and Levitronix CentriMag (Abbott, Santa Clara, CA, USA) assist device implantation for temporary right ventricular support via right femoral vein and an $8 \mathrm{~mm}$ vascular graft anastomosed to the central pulmonary artery were performed. Severe coagulopathy necessitated intraoperative transfusion of 6 units of packed red blood cells, 4 fresh-frozen plasmas, 4 units of platelets, and $2 \mathrm{~g}$ fibrinogen. Despite its potential for serious thromboembolic events [Stokes 2011] recombinant factor VII $(7,000 \mu \mathrm{g})$ also was administered.

Due to ongoing blood loss, the patient required two revisions. Standard surgical measures like additional suturing, pledgeted sutures, fibrin sealants, and other polymeric sealing devices did not provide hemostasis from the bleeding site at the apical inflow cannula. During the third revision, we applied the EVARREST ${ }^{\circledR}$ FSP (Figure 1) around the apex cannula, according to the manufacturer's instructions. (Figure 1)

The bleeding ceased, and the patient was fully anticoagulated, according to LVAD company guidelines without further revisions or blood transfusions. (Figure 2)

\section{DISCUSSION}

Perioperative bleeding is associated with increased mortality and morbidity and leads to acidosis, hemodilution and coagulopathy, making hemostasis even more difficult to achieve [Stokes 2011].

Bleeding is one of the most common complications, following LVAD implantation. Implantation of earlier generation 
pulsatile flow pumps was associated with high rates (up to $50 \%$ ) of bleeding requiring surgical revision [Kasirajan 2000]. The newer generation continuous flow LVADs still have a reoperation rate for bleeding of $30 \%$. Eighty-one percent of these patients required a blood transfusion [Slaughter 2009].

If conventional measures like sutures, clips or electrocautery fail or cannot be used because of anatomical constraints or accessibility like in LVAD surgery, topical absorbable hemostats and recombinant factor VII can be used. If these also fail to achieve hemostasis, the situation becomes desperate.

In this case of troublesome LVAD inflow cannula bleeding, we used fibrin glue along the suture lines during initial LVAD implantation as part of our routine implant protocol. Use of pledgets also was part of our routine implant procedure at that time.

The EVARREST ${ }^{\circledR}$ Fibrin sealant patch is an adjunctive hemostatic patch for use with manual compression. It consists of a flexible matrix of oxidized regenerative cellulose fixed on one side with polyglactin 910 non-woven fibers. It increases the surface area that carries a uniformly imbedded biologic component composed of human fibrinogen and thrombin. When the active side of the patch is exposed to the surface of bleeding tissue, thrombin converts the fibrinogen into fibrin. The result is the formation of a stable blood clot that integrates with the patch's matrix design and sticks to the tissue surface to stop the bleeding.

The efficacy of the EVARREST ${ }^{\circledR}$ FSP has been demonstrated in randomized trials in non-cardiac surgery [Fischer 2013; Koea 2015], but the success rate in cardiac surgery largely is unknown. Corral and coworkers showed that this patch might result in significant hospital cost savings in complicated hepatic and soft tissue surgical bleeding [Corral 2016], if other expensive hemostatic agents like recombinant factor VII can be saved, this is conclusive.

Our case report demonstrates the effectiveness of the EVARREST $^{\circledR}$ FSP as a bail-out hemostatic measure in troublesome LVAD bleeding after failure of standard surgical methods. It has the potential to become is an important adjunct to the cardiac surgeons' armamentarium for intractable bleeding problems in LVAD surgery. Larger studies are required to confirm this finding and possibly extend indications, e.g. toward aortic surgery.

\section{REFERENCES}

Bruckner BA, DiBardino DJ, Ning Q, et al. 2009. High incidence of thromboembolic events in left ventricular assist device patients treated with recombinant activated factor VII. J Heart Lung Transplant. 28: 785-90.

Corral M, Ferko N, Hogan A, et al. 2016. A hospital cost analysis of a fibrin sealant patch in soft tissue and hepatic surgical bleeding. Clinicoecon Outcomes Res. 8: 507-519.

Ding H, Yuan JQ, Zhou JH, et al. 2013. Systematic review and metaanalysis of application of fibrin sealant after liver resection. Curr Med Res Opin. 29: 387-394.

Fischer CP, Bochicchio G, Shen J, et al. 2013. A prospective, randomized, controlled trial of the efficacy and safety of fibrin pad as an adjunct

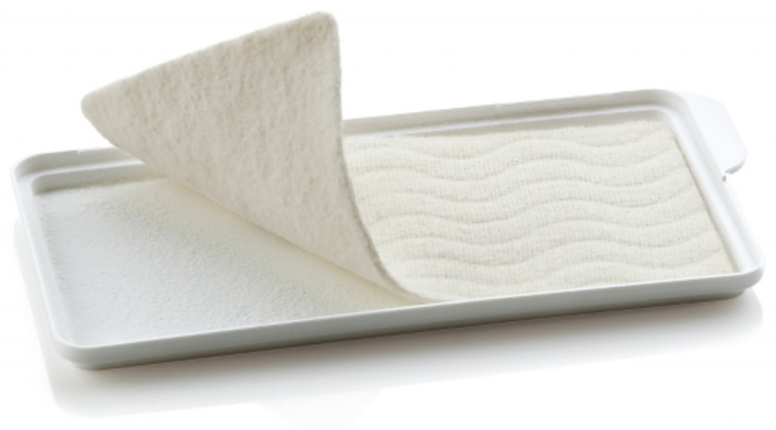

Figure 1. EVARRESTTM - Fibrin Sealant Patch (FSP) (Ethicon, Bridgewater, USA) - courtesy of Ethicon

to control soft tissue bleeding during abdominal, retroperitoneal, pelvic, and thoracic surgery. J Am Coll Surg. 217:385-393.

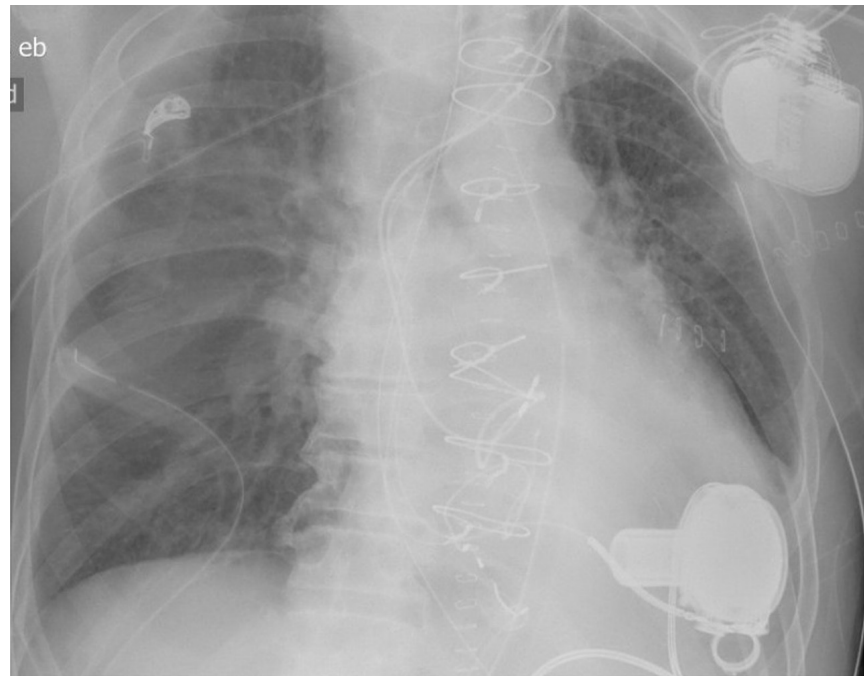

Figure 2. Chest $\mathrm{x}$-ray after third revision with application of EVARREST patch

Kasirajan V, McCarthy PM, Hoercher KJ, et al. 2000. Clinical experience with long-term use of implantable left ventricular assist devices: indications, implantation, and outcomes. Semin Thorac Cardiovasc Surg. 12:229-37.

Koea J, Baldwin P, Shen J, et al. 2015. Safety and hemostatic effectiveness of the fibrin pad for severe soft-tissue bleeding during abdominal, retroperitoneal, pelvic, and thoracic (non-cardiac) surgery: a randomized, controlled, superiority trial. World J Surg. 39: 2663-2669.

Slaughter MS, Rogers JG, Milano CA, et al. 2009. Advanced heart failure treated with continuous-flow left ventricular assist device. N Engl J Med. 361:2241-51.

Stokes ME, Ye X, Shah M, et al. 2011. Impact of bleeding-related complications and/or blood product transfusions on hospital costs in inpatient surgical patients. BMC Health Serv Res. 11:135. 